\title{
Article \\ Potential Instability and Malfunction of Knee Joints with Vastus Medialis Impairment after Total Knee Arthroplasty
}

\author{
Yongkyung Lee ${ }^{1,+}$, Hai-Mi Yang ${ }^{1,2,+}$, Jinju Jang ${ }^{3,4}$, Dai-Soon Kwak ${ }^{5}{ }^{\circledR}$, Jungsung Kim ${ }^{6}$, Heoung-Jae Chun ${ }^{3}$, \\ Dukyoung Jung $7, \ddagger$ and Dohyung Lim ${ }^{1,4, * \mathbb{D}}$
}

1 Department of Mechanical Engineering, Sejong University, Seoul 05006, Korea; dldydrud1634@gmail.com (Y.L.); yhm4956@gmail.com (H.-M.Y.)

2 Cardiovascular and Imaging Devices Division, National Institute of Food and Drug Safety Evaluation, Cheongju 28159, Korea

3 Department of Mechanical Engineering, Yonsei University, Seoul 03722, Korea; jjjang@rnx.re.kr (J.J.); hjchun@yonsei.ac.kr (H.-J.C.)

4 Realization of New X Co., Ltd., Seoul 05006, Korea

5 Department of Anatomy, Catholic Institute for Applied Anatomy, College of Medicine, The Catholic University, Seoul 06591, Korea; daisoon@catholic.ac.kr

6 Department of Research and Development, Corentec Co., Ltd., Seoul 06649, Korea; jskim@corentec.com

7 Seongnam Senior Experience Complex, Eulji University, Seongnam 13135, Korea; dyjung@eulji.ac.kr

* Correspondence: dli349@sejong.ac.kr; Tel.: +82-2-3408-3672

$\dagger$ The first two authors contributed to this article equally.

$\ddagger$ Co-Correspondence author.

Citation: Lee, Y.; Yang, H.-M.; Jang, J.; Kwak, D.-S.; Kim, J.; Chun, H.-J.; Jung, D.; Lim, D. Potential Instability and Malfunction of Knee Joints with Vastus Medialis Impairment after Total Knee Arthroplasty. Appl. Sci. 2021, 11, 2764. https://doi.org/ 10.3390/app11062764

Academic Editor: Rossella Bedini

Received: 3 February 2021

Accepted: 17 March 2021

Published: 19 March 2021

Publisher's Note: MDPI stays neutral with regard to jurisdictional claims in published maps and institutional affiliations.

Copyright: (c) 2021 by the authors. Licensee MDPI, Basel, Switzerland. This article is an open access article distributed under the terms and conditions of the Creative Commons Attribution (CC BY) license (https:// creativecommons.org/licenses/by/ $4.0 /)$.
Abstract: Four pairs of fresh-frozen cadaver knees (eight knees, four male knees) with a mean age of $72 \pm 7$ years were used for tests involving a customized simulator capable of controlling quadriceps loading conditions. The muscle force distribution of the quadriceps for the normal loading condition was applied on the basis of muscle cross-sectional area data, as previously reported (VM: $31 \mathrm{~N}$; RF/VI: $49 \mathrm{~N}$; VL: $45 \mathrm{~N}$ ). To simulate vastus medialis (VM) impairment, we set the muscle force for VM in the muscle force distribution of the quadriceps at zero (VM: $0 \mathrm{~N}$; RF/VI: $49 \mathrm{~N}$; VL: $45 \mathrm{~N}$ ). The joint reaction forces and moments on knee joints that underwent total knee arthroplasty (TKA) did not differ significantly according to VM impairment status for all flexion angles $(p>0.05)$. Nevertheless, the vectors of internal-external moments mostly showed a tendency for alteration from external to internal due to VM impairment. This tendency was evident in 9 cases in 12 total test pairs (with and without VM impairment). Furthermore, the vectors of the anterior-posterior reaction forces mostly showed a tendency to increase anteriorly due to VM impairment. This tendency was also evident in 9 cases in 12 total test pairs (with and without VM impairment). These results indicate that posterior dislocation of the tibia may be induced if VM impairment occurs after TKA. In conclusion, VM impairment in knee joints undergoing TKA may contribute to posterior dislocation of the tibia by a paradoxical roll-back with enhancements of the anterior joint reaction force and external moment during knee-joint flexion. Our findings may be valuable for understanding the mechanism of potential instability and malfunction due to VM impairment in knee joints after TKA, and may help to optimize clinical/rehabilitation training plans to improve the prognosis (stability and function) of knee joints undergoing TKA.

Keywords: total knee arthroplasty; vastus medialis impairment; potential instability; potential malfunction; posterior dislocation

\section{Introduction}

Total knee arthroplasty (TKA) is an orthopedic surgical approach that is widely applied for patients with severely painful joint diseases (e.g., degenerative arthritis, inflammatory arthritis, and avascular necrosis), with a high success rate of $>90 \%$. TKA survival rates reportedly exceed $95 \%$ at 10 years and $90 \%$ at 15 years [1,2]. The Fourth American Joint 
Replacement Registry reported that TKAs were performed for approximately 0.5 million people in the USA in 2016 and will be performed in approximately 3 million people annually worldwide after 2030 [3-5]. However, TKA continues to exhibit failures generally related to pain, infection, osteolysis, wear, aseptic loosening, periprosthetic fracture, instability, or malalignment [5]. Additionally, muscle impairment (weakness) occasionally influences the quality of life after TKA, resulting in direct or indirect TKA failure with adverse prognosis and satisfaction [6-10]. In particular, quadriceps impairment is considered the main type of muscle impairment in patients who have undergone TKA [11-14].

Many researchers have investigated quadriceps impairment in patients who have undergone TKA [11-18]. They identified that the extension moment was generally $20 \%$ lower in knee joints after TKA than in knee joints that had not undergone TKA. Furthermore, Christensen et al. reported that the TKA-related quadriceps impairment produced an asymmetry extension moment, which limited the physical function of the knee joint [3]. Sharkey et al., Cavaignac et al., and Lee et al. have reported that extensor impairment caused by quadriceps impairment led to a greater risk of posterior instability in knee joints that had undergone TKA $[9,19,20]$. Furthermore, Cottino et al. reported that patients with quadriceps impairment had a high risk of hyperextension after TKA because they tended to lock the knee during the extension phase of gait to compensate for a weak extensor mechanism and to avoid instability. This approach led to unreliable results with respect to stability and functional outcomes [8]. Previous clinical studies showed that TKA instability could result from quadriceps impairment. In particular, quadriceps impairment frequently involved the vastus medialis (VM) [21,22]. Quadriceps impairment presumably induces undesirable joint reaction forces and moments due to changes in the force direction and magnitude of the quadriceps in knee joints that have undergone TKA [23,24]. To our knowledge, there is minimal information concerning the mechanisms of instability and malfunction due to VM impairment in knee joints that have undergone TKA.

Changes in the force direction and magnitude of quadriceps by VM impairment may induce changes in the vectors of the joint reaction force and moment, resulting in instability and malfunction within knee joints that have undergone TKA. Thus, the current study was performed to elucidate the mechanisms of potential instability and malfunction due to VM impairment in knee joints that have undergone TKA through analysis of the characteristics of joint reaction forces and moments in knee joints with VM impairment after TKA.

\section{Materials and Methods}

Four pairs of fresh-frozen cadaver knees ( 8 knees, 4 male knees) with a mean age of $72 \pm 7$ years were used in this study. All specimens were macroscopically intact and did not exhibit any gross pathology. After they had been thawed, the cadaveric knees were dissected carefully, while retaining the femur, tibia, patella, ligament, patellar tendon, quadriceps, skin, and subcutaneous fat surrounding the knee joint. The femur and tibia were cut at $29 \mathrm{~cm}$ from the knee joint line. The quadriceps were separated into individual components: rectus femoris (RF), vastus intermedius (VI), vastus lateralis $(\mathrm{VL})$, and VM. This separation was performed with a guide using fascial planes between muscles. For each cadaver, one knee was randomly assigned to undergo TKA and the other was assigned to a control condition group that did not undergo TKA. All TKA surgical procedures were performed by a single surgeon using a standard cruciate-retaining prosthesis instrumentation system (LOSPA; Corentec Co. Ltd., Seoul, Korea) with a highconformity tibial insert design.

Both the femur and tibia were secured within cylinder tubing $(3.5 \mathrm{~cm}$ diameter and $9 \mathrm{~cm}$ height) filled with resin (Illinois Tool Works Inc., Cincinnati, OH, USA). The femur and tibia were fixed to the knee simulator system in a state of full knee-joint extension. The knee-simulator system can provide 4 degrees of freedom at the femur and the tibia jigs (Figure 1). However, in the current study, the simulator system provided 2 degrees of freedom at the femur and one degree of freedom at the tibia (Figure 2). Flexion motion was controlled by lowering the crossbar on the knee-simulator system to induce rotation of the 
femur jig and tibia jig at $3^{\circ} / \mathrm{s}$. This was driven by a linear actuator at a speed of $40 \mathrm{~mm} / \mathrm{s}$. The reaction forces and moments in the knee joint were quantified through a 6-axis load cell with a sampling frequency of $1000 \mathrm{~Hz}$ (Advanced Mechanical Technology Inc., Watertown, MA, USA) mounted on the bottom of the tibia jig of the simulator system (Figure 2). The coordinate system of the knee joint was defined as the $x$-axis in the anterior/posterior direction, $y$-axis in the medial/lateral direction, and $z$-axis in the proximal/distal direction (Figure 2). The knee flexion angle was then measured continuously from $0^{\circ}$ to $90^{\circ}$, using 2 inertial measurement unit sensors (SEED Tech Co., Ltd., Bucheon, Korea) attached to the posterior-proximal position of the femur and the posterior-distal position of the tibia (Figure 2). The coordinate system of the inertial measurement unit sensors at the femur and tibia were defined as the $x$-axis in the medial-lateral direction, $y$-axis in the anteriorposterior direction, and $z$-axis in the proximal-distal direction (Figure 2).

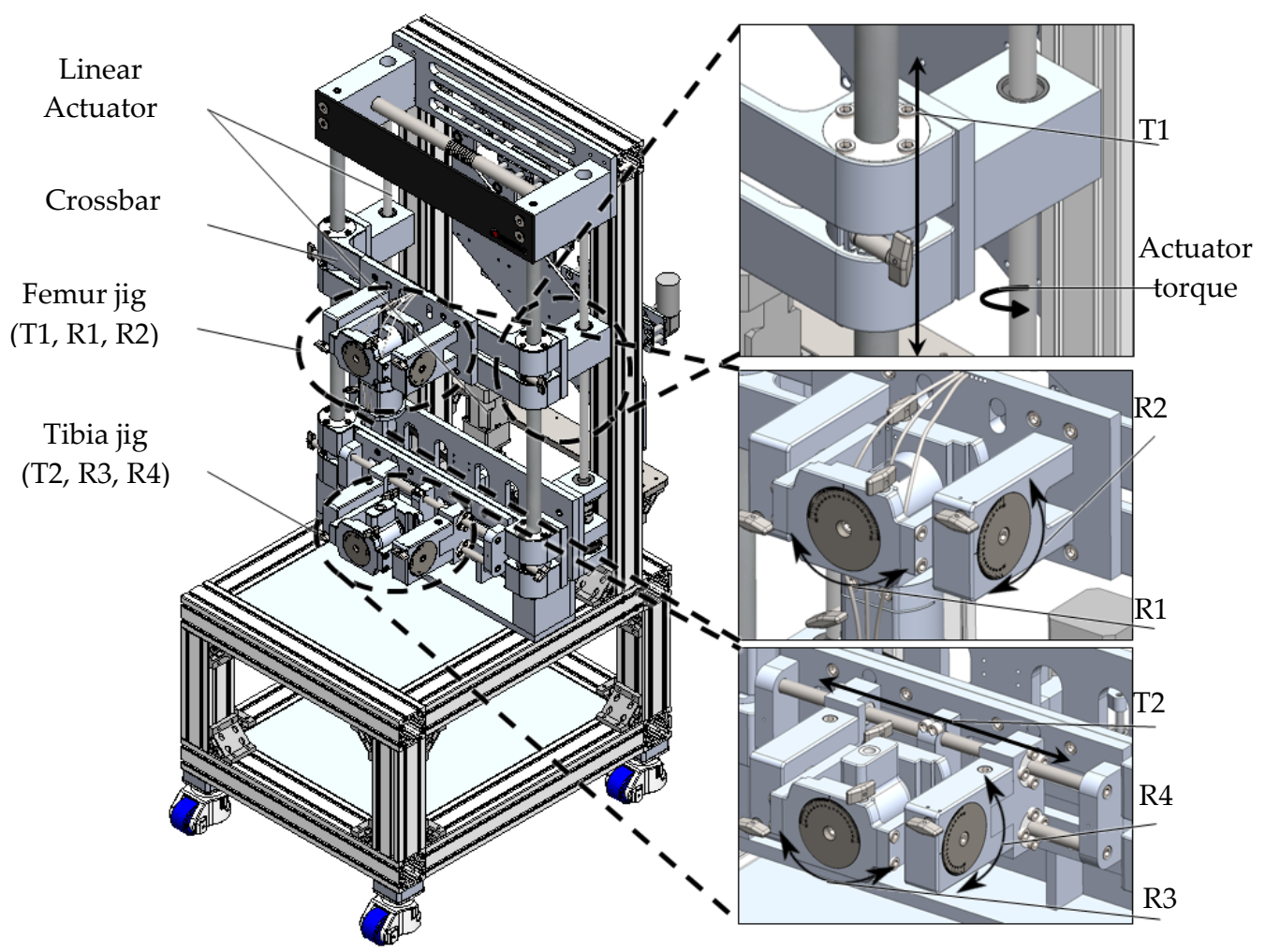

Figure 1. Schematic overview of simulator components and their degrees of freedom (T1: proximal-distal displacement on the femur; R1: varus-valgus rotation on the femur; R2: flexion-extension rotation on the femur; T2: medial-lateral displacement on the tibia; R3: varus-valgus rotation on the tibia; R4: flexion-extension rotation on the tibia) in the knee-simulator system.

Each quadriceps muscle (RF/VI, VM, and VL) was clamped and connected to loading weights through a wire loop. The muscle force distribution of the quadriceps for the normal loading condition was applied on the basis of muscle cross-sectional area data, as previously reported (VM: 31 N; RF/VI: 49 N; VL: 45 N) [25-30]. To simulate VM impairment, we set the muscle force for $\mathrm{VM}$ in the muscle force distribution of the quadriceps to zero (VM: $0 \mathrm{~N}$; RF/VI: $49 \mathrm{~N}$; VL: $45 \mathrm{~N}$ ). The force directions were adjusted in accordance with the primary fiber direction of respective musculature $[25,27]$. The force direction of RF/VI was set equal to the femoral axis in the frontal plane. In the sagittal plane, the RF/VI was then placed at an angle of $16.8^{\circ} \pm 1.3^{\circ}$ anterior to the femoral axis. The force directions of the $\mathrm{VM}$ and VL were placed at angles of $28.9^{\circ} \pm 0.7^{\circ}$ and $24.5^{\circ} \pm 2.0^{\circ}$ relative to the femoral axis in the frontal plane, and $13.6^{\circ} \pm 1.2^{\circ}$ posterior to the femoral axis in the sagittal plane. The test for each condition was then repeated 3 times to confirm reproducibility. Table 1 summarizes all test conditions and total numbers of tests. 


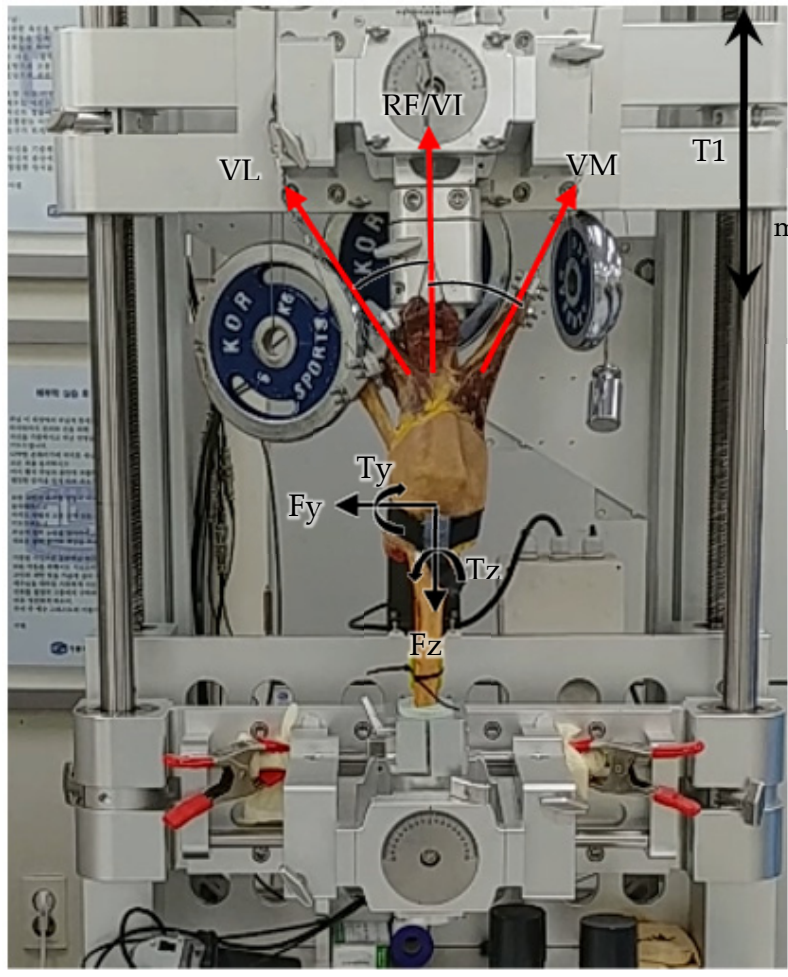

(a)
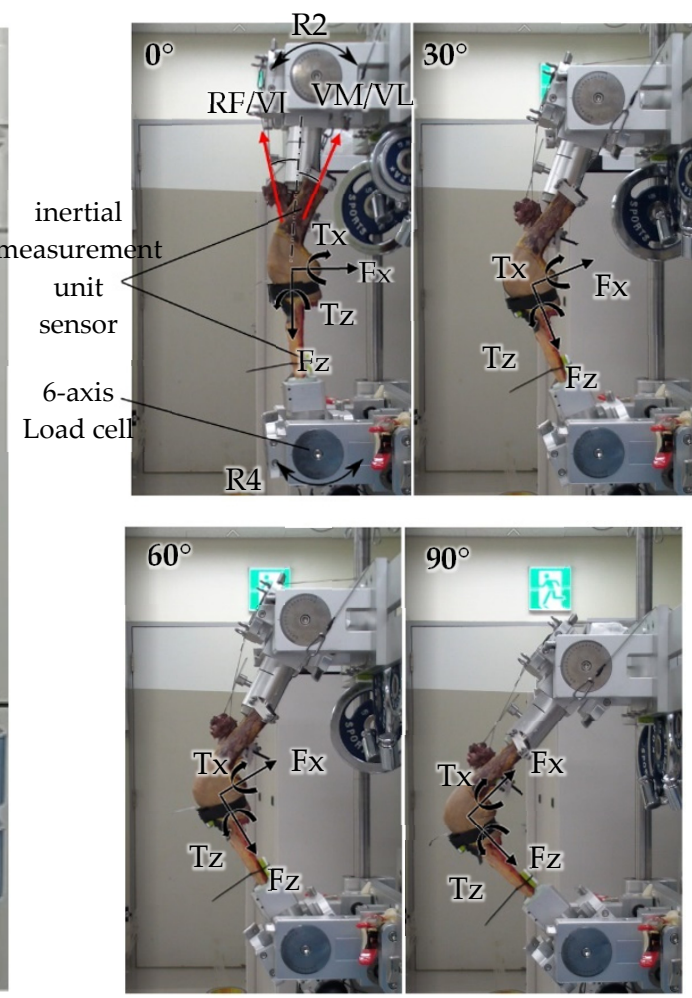

(b)

Figure 2. Test configurations for muscle forces and directions (RF: rectus femoris; VI: vastus intermedius; VL: vastus lateralis; VM: vastus medialis) applied to the knee joint and the coordinate system (Fx: anterior-posterior reaction force; Fy: medial-lateral reaction force; Fz: proximal-distal reaction force; Tx: varus-valgus moment; Ty: flexion-extension moment; Tz: internal-external moment) on the knee joint and their degrees of freedom (T1: proximal-distal displacement on the femur; R2: flexion-extension rotation on the femur; R4: flexion-extension rotation on the tibia): (a) frontal view; (b) sagittal view for each flexion angle $\left(0^{\circ}, 30^{\circ}, 60^{\circ}\right.$, and $\left.90^{\circ}\right)$.

Table 1. Test conditions and total test count performed in the current study.

\begin{tabular}{cccc}
\hline & VM Impairment & Repetition Count & Total Test Count $(\mathbf{m} * \mathbf{n})$ \\
\hline $\begin{array}{c}\text { TKA not performed } \\
\left(\mathrm{m}^{1}=4\right)\end{array}$ & With $^{3}\left(\mathrm{~m}^{1}=4\right)$ & $\mathrm{n}^{2}=3$ & 12 \\
Without $^{4}\left(\mathrm{~m}^{1}=4\right)$ & $\mathrm{n}^{2}=3$ & 12 \\
\hline TKA performed & With $^{3}\left(\mathrm{~m}^{1}=4\right)$ & $\mathrm{n}^{2}=3$ & 12 \\
$\left(\mathrm{~m}^{1}=4\right)$ & Without $^{4}\left(\mathrm{~m}^{1}=4\right)$ & $\mathrm{n}^{2}=3$ & 12 \\
\hline
\end{tabular}

${ }^{1} \mathrm{~m}=$ number of specimens. ${ }^{2} \mathrm{n}=$ repetition count of the test for each specimen. ${ }^{3}$ Vastus medialis (VM) muscle force was set to zero (VM: $0 \mathrm{~N}$; RF/VI: $49 \mathrm{~N}$; VL: $45 \mathrm{~N}$ ). ${ }^{4} \mathrm{VM}$ muscle force was set to normal muscle force (VM: 31 N; RF/VI: 49 N; VL: 45 N).

A paired Student's $t$-test was used to identify the difference between the knee joint response (reaction forces and moments) with/without TKA on quadriceps with/without VM impairment. Equal variance was verified by Levene's test, and $p<0.05$ was taken to indicate statistical significance.

\section{Results}

The reaction forces and moments of knee joints that did not undergo TKA and did or did not exhibit VM impairment for the flexion angle are shown in Figure 3. Within $0-30^{\circ}$ flexion angles, the dominant vector of the medial-lateral reaction forces was altered from lateral to medial secondary to VM impairment $(p<0.05)$. Here, the medial-lateral reaction forces on quadriceps with/without VM impairment were significantly different 
at $10^{\circ}(13.8 \pm 3.0 \mathrm{~N})$ and $20^{\circ}(9.2 \pm 0.2 \mathrm{~N})$ flexion angles $(p<0.05)$. The other reaction forces and moments with/without VM impairment were not significantly different $(p>$ 0.05). Nevertheless, the vectors of the varus-valgus and internal-external moments showed tendencies for alteration from valgus and external to varus and internal. These tendencies were evident in 6 cases in 12 total test pairs (with and without VM impairment). Within $40-90^{\circ}$ flexion angles, all reaction forces and moments with/without VM impairment were not significantly different $(p>0.05)$. For knee joints that had undergone TKA, the joint reaction forces and moments are shown in Figure 4. The joint reaction forces and moments with/without VM impairment were not significantly different for all flexion angles $(p>$ $0.05)$. Nevertheless, the vectors of internal-external moments mostly showed a tendency for alteration from external to internal due to VM impairment. This tendency was evident in 9 cases in 12 total test pairs (with and without VM impairment) (Figure A1). Furthermore, the vectors of the anterior-posterior reaction forces mostly showed a tendency for anterior increase due to VM impairment. This tendency was evident in 9 cases in 12 total test pairs (with and without VM impairment) (Figure A1).

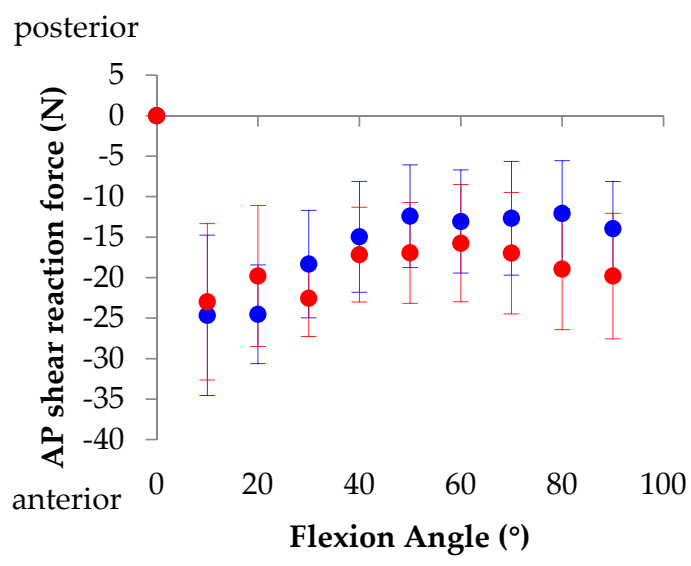

(a)

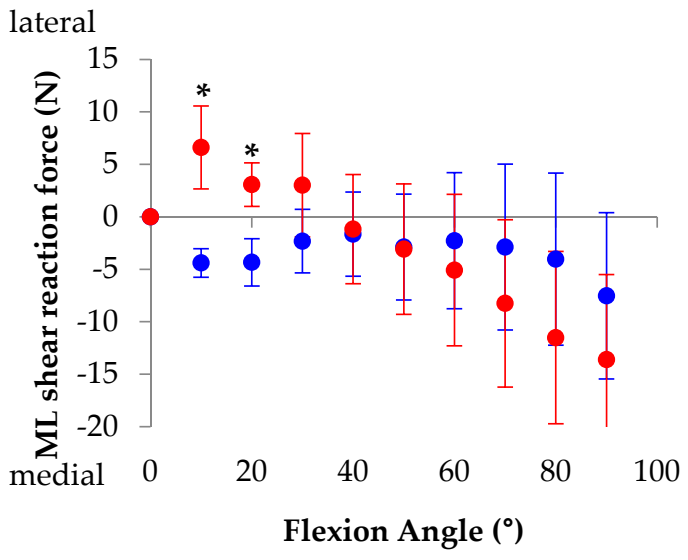

(c)

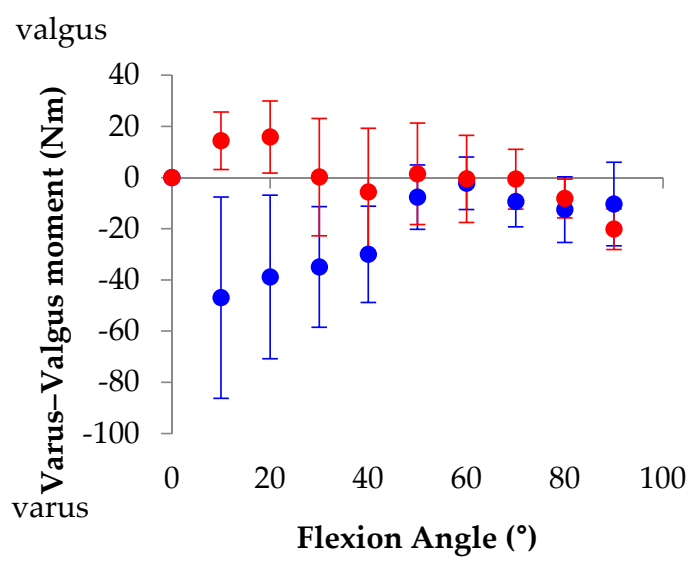

(b)

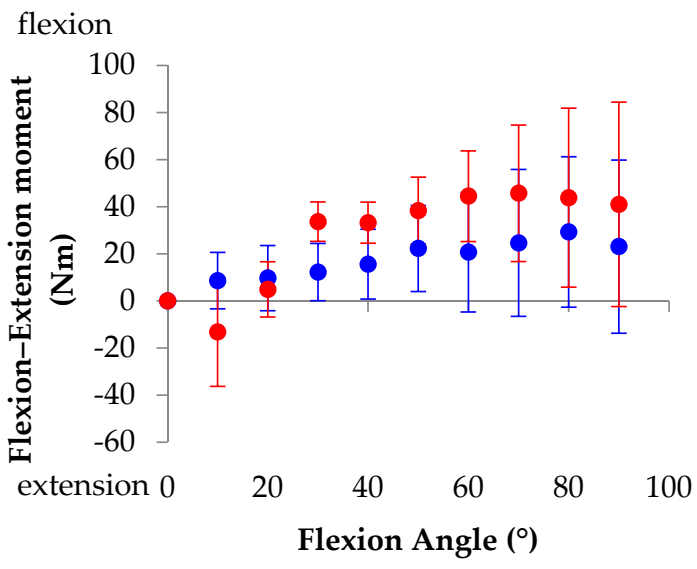

(d)

Figure 3. Cont. 


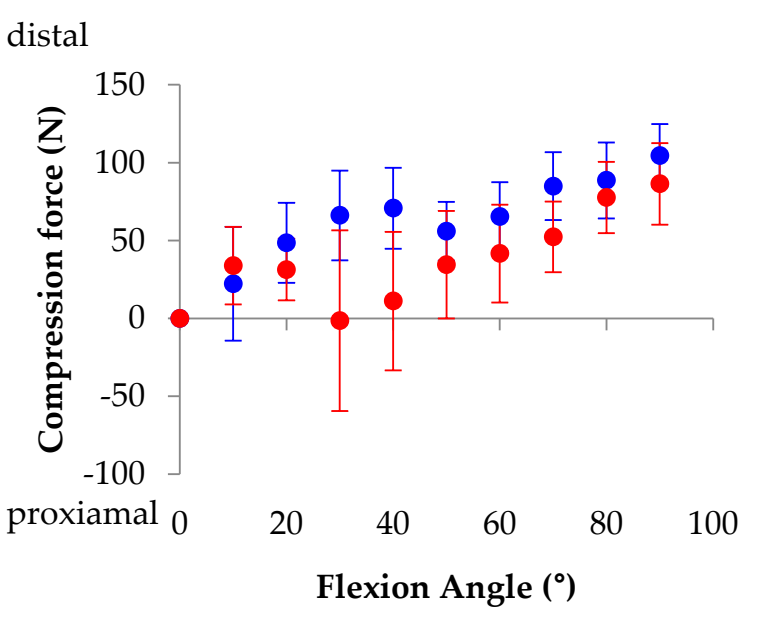

(e) external

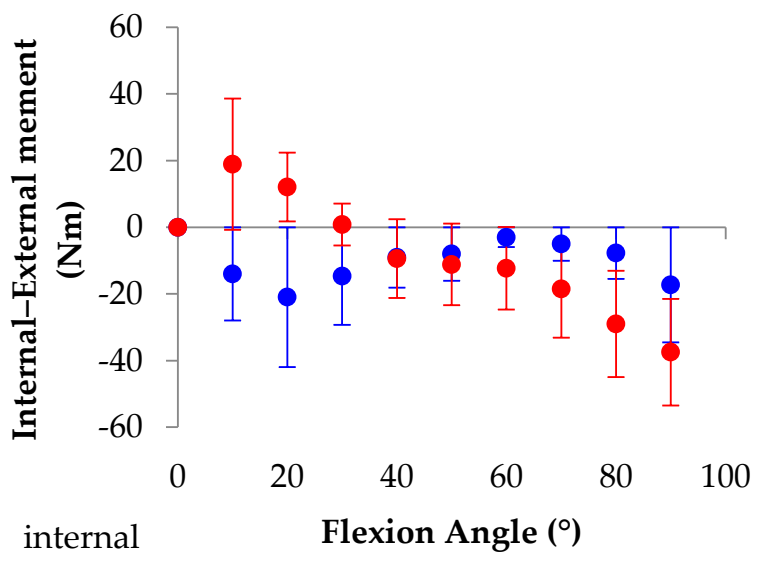

(f)

- Native knee joint (without impariment) • Native knee joint (with VM impairment)

Figure 3. Knee-joint reaction forces and moments in native knee joints with/without VM impairment during knee-joint flexion: (a) anterior-posterior reaction force (Fx); (b) varus-valgus moment (Tx); (c) medial-lateral reaction force (Fy); (d) flexion-extension moment (Ty); (e) proximal-distal reaction force (Fz); (f) internal-external moment (Tz). ${ }^{*} p<0.05$.

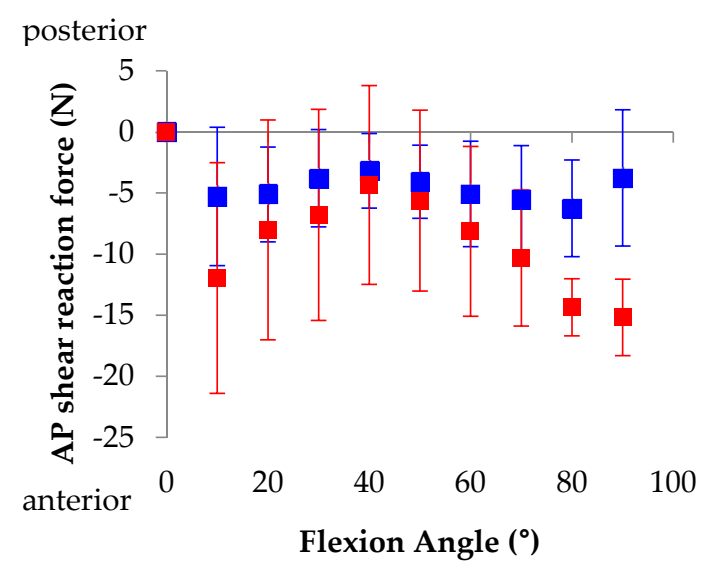

(a)

lateral

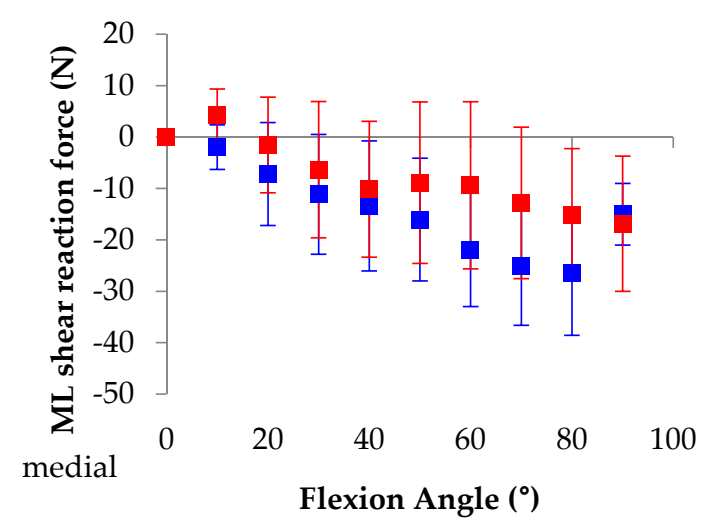

(c)

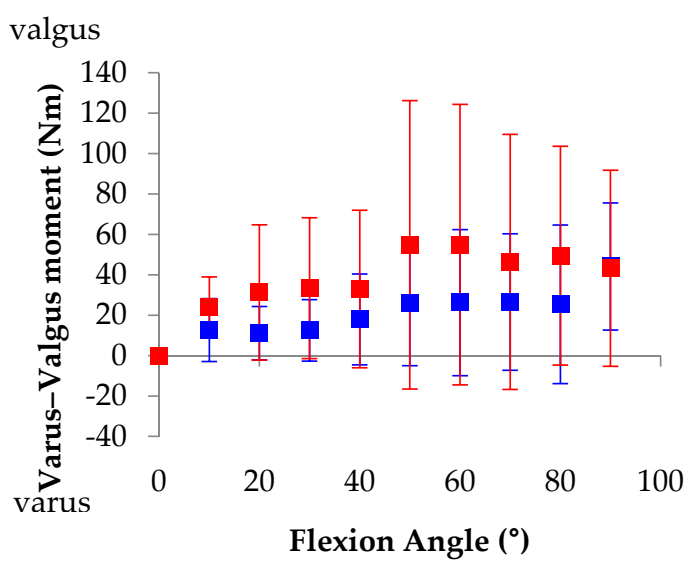

(b)

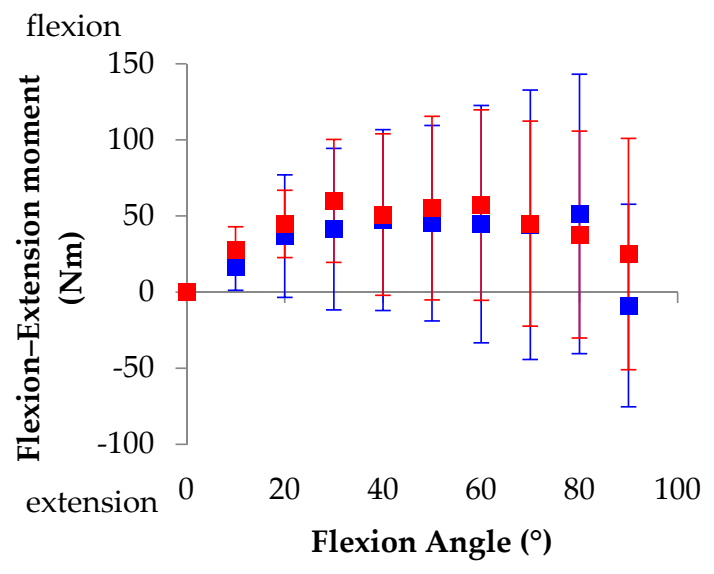

(d)

Figure 4. Cont. 


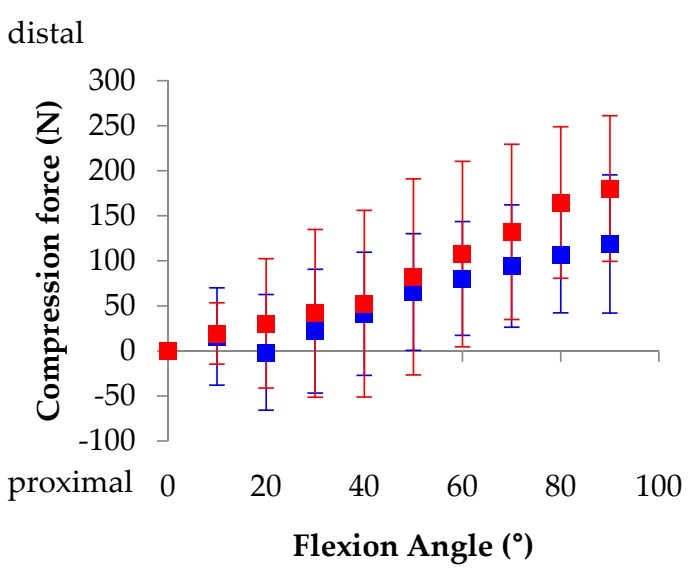

(e)

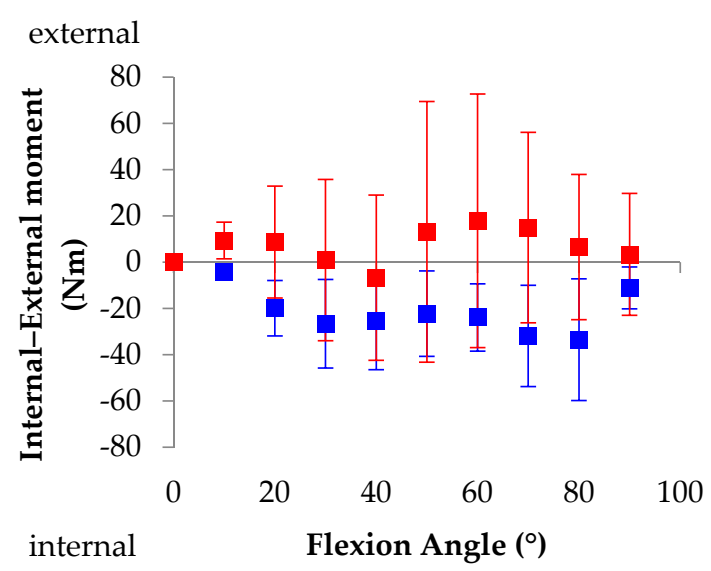

(f)

- Knee joint operated TKA (without VM impairment) - Knee joint operated TKA (with VM impairment)

Figure 4. Knee-joint reaction forces and moments in knee joints that underwent total knee arthroplasty (TKA) with/without VM impairment during knee-joint flexion: (a) anterior-posterior reaction force (Fx); (b) varus-valgus moment (Tx); (c) medial-lateral reaction force (Fy); (d) flexion-extension moment (Ty); (e) proximal-distal reaction force (Fz); (f) internalexternal moment $(\mathrm{Tz})$.

\section{Discussion and Conclusions}

Quadriceps impairment reportedly induces occasional inability to fully activate the quadriceps following TKA due to voluntary quadriceps contraction disability [11-18], resulting in hyperextension and posterior dislocation of the tibia [3,6-8,14,17]. Although rehabilitation exercises are generally prescribed to enhance the activation of the quadriceps following TKA $[17,31-35]$, the fundamental clinical problems have not been resolved. Therefore, greater clarity is needed concerning the biomechanical mechanisms that cause hyperextension and posterior dislocation of the tibia due to quadriceps impairment. To our knowledge, previous studies have provided limited confirmation of alterations in the flexion-extension moment, despite alterations in all knee-joint reaction forces and moments [11-18]. Overall, there is a need to confirm the characteristics of alterations in all knee-joint reaction forces and moments because impairment in VM, a component of the quadriceps, affects the muscle force vector. Accordingly, if alterations in all knee-joint reaction forces and moments appear repeatedly, they affect the surrounding ligaments and tendons that balance knee-joint motion, resulting in knee-joint instability (i.e., hyperextension and posterior dislocation of the tibia in knee joints that have undergone TKA). Therefore, the current study investigated the characteristics of alterations in all knee-joint reaction forces and moments due to VM impairment and identified tendencies for changes in the vector directions of specific joint reaction forces and moments. The results indicate that changes in the vector directions of specific joint reaction forces and moments due to VM impairment may serve as undesirable kinematic mechanisms in the knee joint, which can induce posterior dislocation of the tibia. Our findings may offer mechanistic insights concerning posterior dislocation of the tibia from quadriceps impairment following TKA.

It is important to control the quadriceps contraction forces when identifying the mechanisms of potential joint instability and malfunction due to VM impairment in knee joints that have undergone TKA. In this study, hamstring measurements were not considered because the quadriceps produces a comparatively greater contraction force [36]. Therefore, we presumed that a specific focus on the quadriceps was more appropriate. The normal quadriceps contraction forces were established on the basis of the contraction ratio loaded on each component of the quadriceps, in accordance with the observation by Wickiewicz et al. that the muscle cross-sectional area is generally proportional to the muscle contraction force [28]. However, the quadriceps contraction forces in the VM impairment 
condition were established with the assumption that no contraction force acted on the $\mathrm{VM}$, because no quadriceps contraction ratio has been reported for the VM impairment condition. Although it is difficult to determine whether the VM completely contracts in the VM impairment condition, VM contraction is presumed to function incorrectly because VM impairment is reportedly caused by a voluntary contraction disorder related to neuromuscular inhibition $[21,23,34]$. Furthermore, muscle force adjustment can complement the function of VM by strengthening muscles around the knee joint during the onset of VM impairment. The muscle force adjustment may exhibit greater alterations in the direction of muscle force, and thus it is likely to substantially influence the knee-joint reaction forces and moments. However, it is difficult to estimate the required muscle adjustment and the necessary extent of compensatory contraction force. Thus, there is a limit to the application of the accurate quadriceps contraction ratio in the VM impairment condition. Removing only the load applied to the VM is presumably the worst situation for VM impairment that can be achieved in practice. Additionally, characteristics of the alterations of the knee-joint reaction forces and moments are presumed to be clearly visible when the worst situation is applied, and the biomechanical mechanism of TKA instability due to VM impairment can be clearly confirmed.

Kwak et al. [37] and Bull et al. [38] reported that anterior translation was enhanced up to low knee-joint flexion and reduced thereafter in the native knee joint, which is consistent with our findings. Enhancements of the anterior translation and reaction force within $0-20^{\circ}$ of knee-joint flexion occur because the direction of the patellar tendon load transmitted from the quadriceps is gradually shifted from the anterior to the proximal direction during low knee-joint flexion [29,39]. Kwak et al. [37] reported that the tibia was translated medially and rotated in the varus and internal directions as the knee joint flexed in the native knee joint, which is consistent with our findings. In particular, internal rotation occurs at $0-30^{\circ}$ flexion by screw home rotation of the knee, which we have identified as internal moment. Additionally consistent with our findings, Zaggagnini et al. [40], Wunschel et al. [41], and Akbari et al. [42] showed that tendencies of the anterior, medial translation, and internal rotation directions were similar in knee joints that had undergone TKA and did not exhibit VM impairment when compared with those of the native knee joint. However, the current study confirmed that the valgus moment generally occurred in knee joints that had undergone TKA, in contrast to the findings of previous studies $[38,41,43,44]$. Bull et al. [38] and Wunschel et al. [41] reported that a varus moment had occurred (as in previous studies [43,44]), but Akbari et al. [42] confirmed that a valgus moment was present in knee joints that underwent TKA. These findings indicate that varus-valgus moments may vary according to TKA design and the surgeon's surgical methodology and technique. Accordingly, our experimental protocol and results are largely adequate and may be reliable for assessing the aim of the current study.

In this study, we showed that knee joints with VM impairment generated a lateral reaction force and valgus/external moment that differed from those of the native knee joint within $10-20^{\circ}$ knee-joint flexion. This result was likely caused by the lateral translation of the contraction force of the quadriceps due to VM impairment (Figure 5). Knee joints that underwent TKA showed that the joint reaction forces and moments tended to change from internal to external moments due to VM impairment. In particular, knees that had undergone TKA and exhibited VM impairment showed a sharp enhancement of the anterior joint reaction force within $40-90^{\circ}$ knee-joint flexion, in contrast to knee joints that had not undergone TKA but exhibited VM impairment. These results might have been caused by alteration of the degree of resistance to the anterior reaction force due to anterior cruciate ligament removal in the TKA operation, as well as the restriction of roll-back motion during knee-joint flexion. Roll-back of the knee joint is reportedly induced by moving the contact point of the femur and tibia in the posterior direction by internal rotation of the tibia and posterior translation of the femur. However, VM impairment in knee joints that have undergone TKA may weaken the posterior force applied to the femur through the patella and restrict both the posterior translation of the femur and internal rotation of the tibia 
by inducing the patellar tendon to pull the tibia in the direction of the external rotation, as shown in Figure 5. Finally, VM impairment may cause a paradoxical roll-back motion during knee-joint flexion, resulting in a greater risk of posterior dislocation of the tibia.

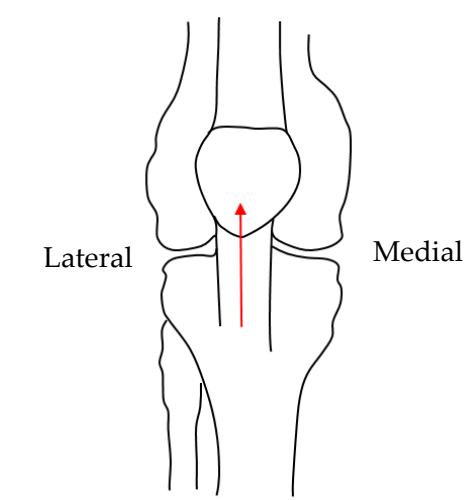

Knee joint without VM impairment

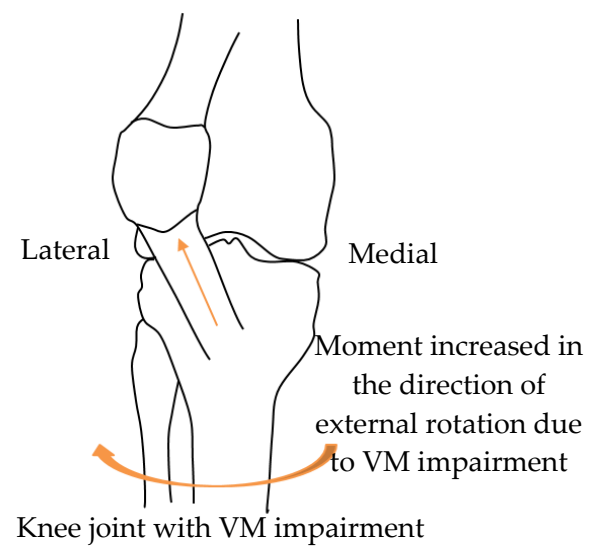

(a)

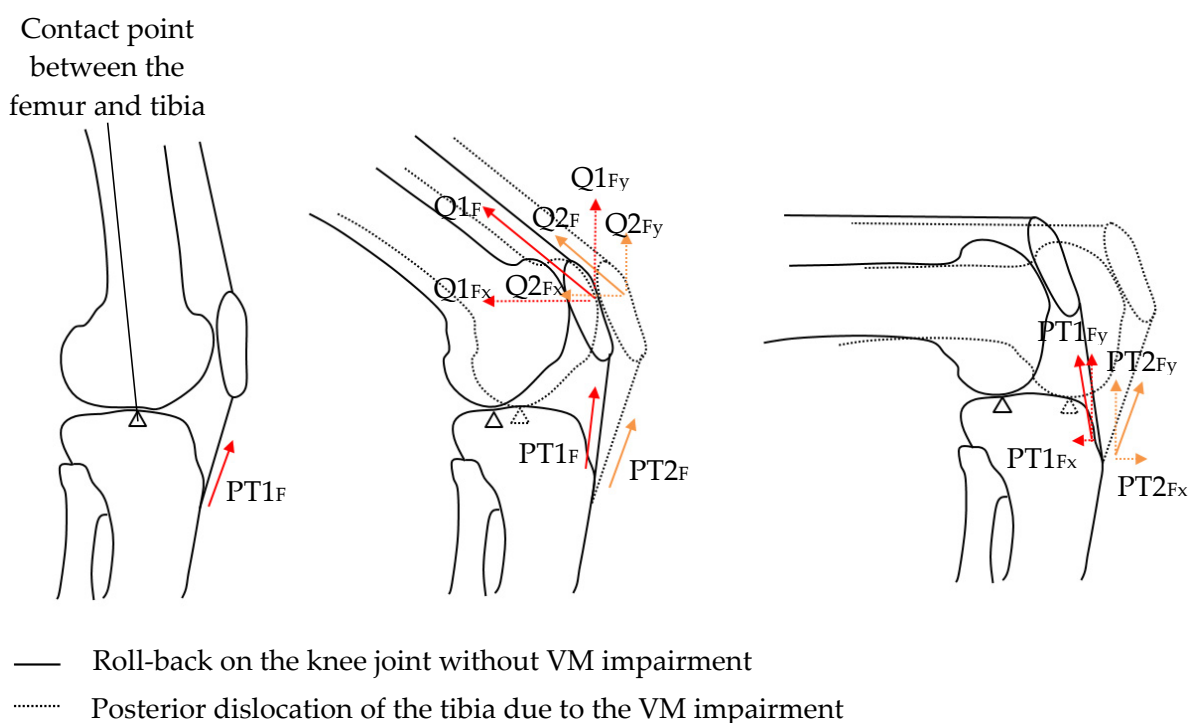

(b)

Figure 5. Changes in quadriceps and patellar tendon vectors of the load (Q1F: quadriceps force without VM impairment; Q2F: quadriceps force with VM impairment; PT1F: patellar tendon force without VM impairment; PT2F: patellar tendon force with VM impairment) due to VM impairment: (a) increased external moment due to VM impairment on the frontal plane; (b) posterior dislocation of the tibia due to VM impairment on the sagittal plane.

Although there were no significant changes in the joint reaction forces and moments due to VM impairment for knee joints with/without TKA, there was a change from valgus and external to varus and internal due to VM impairment on the native knee joint. In this report, the change was observed in $50 \%$ of cases (six cases) due to VM impairment. In addition, we observed increases in the external moment and the anterior joint reaction force due to VM impairment on the knee joint that underwent TKA. Here, $80 \%$ of cases (nine cases) showed increases due to VM impairment. Therefore, posterior dislocation of the tibia may be induced if VM impairment occurs after TKA. This is consistent with clinical reports showing that posterior dislocations of the tibia have been reported in patients with VM impairment, particularly quadriceps impairment, following TKA in clinical practice $[9,19,20]$. 
This study had some limitations. First, it was not possible to change quadriceps contraction during flexion of the knee. In general, the quadriceps contraction force changes during flexion. However, in this study, our technology was limited in its ability to simultaneously change each of the VM, VL, and RF/VI at the same rate. Therefore, we presume that by overcoming the technical limitation of simultaneously and continuously changing the same ratio or desired contractile forces in each muscle, we will obtain results more similar to clinical findings. Second, motion analysis of the knee joint could not be performed concurrently with measurements of joint reaction forces and moments. To obtain the joint reaction forces and moments in a six-axis load cell, we were required to restrain many degrees of freedom of the tibia, which limited measurement of the change in motion. In a future study, we plan to consider simultaneous motion analysis and measurement of the joint reaction forces and moments after finding a method that can be applied to the knee with six degrees of freedom. This will aid in reliable identification of the mechanisms of potential instability and malfunction due to VM impairment after TKA.

In conclusion, we showed that VM impairment in the knee joint after TKA may be associated with posterior dislocation of the tibia by a paradoxical roll-back with increases in the anterior joint reaction force and the external moment during knee joint flexion. This increases our understanding of the mechanism of potential instability and malfunction due to VM impairment on the knee joint after TKA, and can be used to optimize clini$\mathrm{cal} /$ rehabilitation training plans for improving the prognosis (stability and function) of the knee joint after TKA.

Author Contributions: Conceptualization, D.L.; methodology, Y.L., H.-M.Y., J.J., D.-S.K.; investigation, Y.L., H.-M.Y., J.J.; writing-original draft preparation, Y.L., H.-M.Y.; writing-review and editing, J.K., H.J.C., D.J., D.L.; supervision, D.L. All authors have read and agreed to the published version of the manuscript.

Funding: National Research Foundation of Korea and Korea Medical Device Development Fund.

Institutional Review Board Statement: Not applicable.

Acknowledgments: This work was supported by the National Research Foundation of Korea (NRF) grant funded by the Korean government (MSIP) (NRF-2017M3A9E9073545) and the Korea Medical Device Development Fund grant funded by the Korean government (the Ministry of Science and ICT; the Ministry of Trade, Industry and Energy; the Ministry of Health and Welfare; the Ministry of Food and Drug Safety) (project number: 202011B32).

Conflicts of Interest: The authors declare no conflict of interest.

\section{Appendix A}

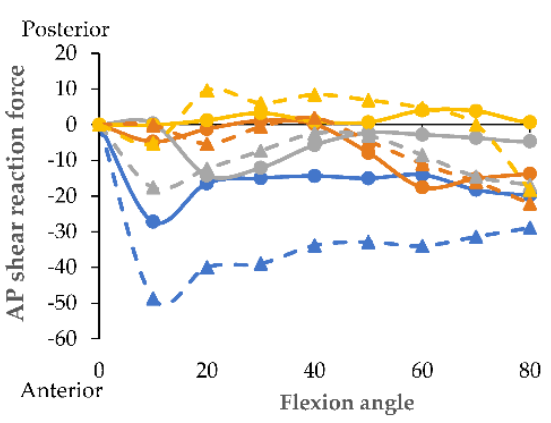

(a)

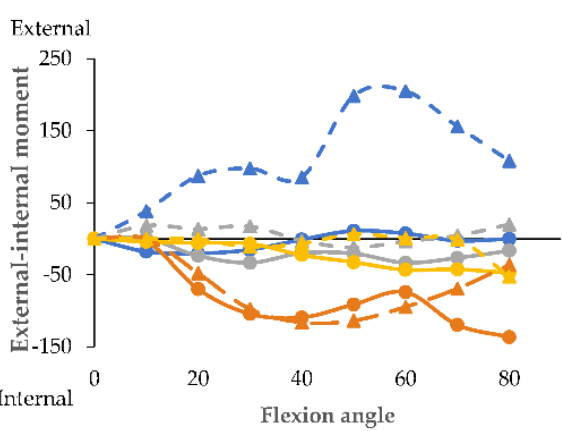

(b)

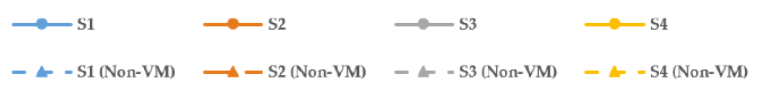

Figure A1. Knee-joint reaction forces and moments in each knee joint that underwent TKA with/without VM impairment (Sn: specimen number, non-VM: with VM impairment) during knee-joint flexion: (a) anterior-posterior reaction force (Fx); (b) internal-external moment (Tz). 


\section{References}

1. Oliver, W.M.; Arthur, C.H.; Wood, A.M.; Clayton, R.A.; Brenkel, I.J.; Walmsley, P. Excellent survival and good outcomes at 15 years using the press-fit condylar sigma total knee arthroplasty. J. Arthroplast. 2018, 33, 2524-2529. [CrossRef]

2. Putman, S.; Argenson, J.-N.; Bonnevialle, P.; Ehlinger, M.; Vie, P.; Leclercq, S.; Bizot, P.; Lustig, S.; Parratte, S.; Ramdane, N.J.O.; et al. Ten-year survival and complications of total knee arthroplasty for osteoarthritis secondary to trauma or surgery: A French multicentre study of 263 patients. Orthop. Traumatol. Surg. Res. 2018, 104, 161-164. [CrossRef] [PubMed]

3. Christensen, J.C.; Mizner, R.L.; Foreman, K.B.; Marcus, R.L.; Pelt, C.E.; LaStayo, P.C. Quadriceps weakness preferentially predicts detrimental gait compensations among common impairments after total knee arthroplasty. J. Orthop. Res. 2018, 36, $2355-2363$. [CrossRef] [PubMed]

4. Berry, D.J. Fourth AJRR Annual Report on Hip and Knee Arthroplasty Data; American Joint Replacement Registry: Rosemont, IL, USA, 2017.

5. Pitta, M.; Esposito, C.I.; Li, Z.; Lee, Y.Y.; Wright, T.M.; Padgett, D.E. Failure after modern total knee arthroplasty: A prospective study of 18,065 knees. J. Arthroplast. 2018, 33, 407-414. [CrossRef] [PubMed]

6. Mullaji, A.B.; Shetty, G.M. The Unstable Knee. In Deformity Correction in Total Knee Arthroplasty; Springer: Berlin/Heidelberg, Germany, 2014; pp. 129-138.

7. Arumilli, B.R.B.; Ferns, B.; Smith, M.; Thalava, R.; Obeid, E.; Muddu, B. Non-traumatic dislocation (Cam Jump) in a revision knee: A case report. Cases J. 2009, 2, 7001. [CrossRef]

8. Cottino, U.; Sculco, P.K.; Sierra, R.J.; Abdel, M.P. Instability after total knee arthroplasty. Orthop. Clin. 2016, 47, 311-316. [CrossRef]

9. Lee, H.M.; Kim, J.P.; Chung, P.H.; Kang, S.; Kim, Y.S.; Go, B.S. Posterior dislocation following revision total knee replacement arthroplasty: A case report and literature analysis. Eur. J. Orthop. Surg. Traumatol. 2018, 28, 1641-1644. [CrossRef]

10. Parween, R.; Shriram, D.; Mohan, R.E.; Lee, Y.H.D.; Subburaj, K. Method for evaluating effects of unloader knee braces on joint health: A review. Biomed. Eng. Lett. 2019, 9, 153-168. [CrossRef]

11. Berman, A.T.; Bosacco, S.J.; Israelite, C. Evaluation of total knee arthroplasty using isokinetic testing. Clin. Orthop. Relat. Res. 1991, 271, 106-113. [CrossRef]

12. Lorentzen, J.S.; Petersen, M.M.; Brot, C.; Madsen, O.R. Early changes in muscle strength after total knee arthroplasty: A 6-month follow-up of 30 knees. Acta Orthop. Scand. 1999, 70, 176-179. [CrossRef]

13. Rodgers, J.A.; Garvin, K.L.; Walker, C.W.; Morford, D.; Urban, J.; Bedard, J. Preoperative physical therapy in primary total knee arthroplasty. J. Arthroplast. 1998, 13, 414-421. [CrossRef]

14. Walsh, M.; Woodhouse, L.J.; Thomas, S.G.; Finch, E. Physical impairments and functional limitations: A comparison of individuals 1 year after total knee arthroplasty with control subjects. Phys. Ther. 1998, 78, 248-258. [CrossRef]

15. Berth, A.; Urbach, D.; Awiszus, F. Improvement of voluntary quadriceps muscle activation after total knee arthroplasty. Arch. Phys. Med. Rehabil. 2002, 83, 1432-1436. [CrossRef] [PubMed]

16. Huang, C.H.; Cheng, C.K.; Lee, Y.T.; Lee, K.S. Muscle strength after successful total knee replacement: A 6-to 13-year followup. Clin. Orthop. Relat. Res. 1996, 328, 147-154. [CrossRef]

17. Mizner, R.L.; Petterson, S.C.; Snyder-Mackler, L. Quadriceps strength and the time course of functional recovery after total knee arthroplasty. J. Orthop. Sports Phys. Ther. 2005, 35, 424-436. [CrossRef]

18. Silva, M.; Shepherd, E.F.; Jackson, W.O.; Pratt, J.A.; McClung, C.D.; Schmalzried, T.P. Knee strength after total knee arthroplasty. J. Arthroplast. 2003, 18, 605-611. [CrossRef]

19. Cavaignac, E.; Tricoire, J.; Pailhé, R.; Murgier, J.; Reina, N.; Chiron, P.; Laffosse, J.M. Recurring intraprosthetic dislocation of rotating-hinge total knee prosthesis. Effect of implant design on intrinsic stability. Orthop. Traumatol. Surg. Res. 2014, 100, 835-837. [CrossRef] [PubMed]

20. Sharkey, P.F.; Hozack, W.J.; Booth, J.R.; Balderston, R.A.; Rothman, R.H. Posterior dislocation of total knee arthroplasty. Clin. Orthop. Relat. Res. 1992, 278, 128-133. [CrossRef]

21. Fitzgerald, G.K. Therapeutic exercise for knee osteoarthritis: Considering factors that may influence outcome. Eur. Med. 2005, 41, 163.

22. Bolgla, L.A.; Shaffer, S.W.; Malone, T.R. Vastus medialis activation during knee extension exercises: Evidence for exercise prescription. J. Sport Rehabil. 2008, 17, 1-10. [CrossRef]

23. Mizner, R.L.; Petterson, S.C.; Stevens, J.E.; Vandenborne, K.; Snyder-Mackler, L. Early quadriceps strength loss after total knee arthroplasty: The contributions of muscle atrophy and failure of voluntary muscle activation. J. Bone Jt. Surg. Am. 2005, 87, 1047. [CrossRef] [PubMed]

24. Sakai, N.; Luo, Z.P.; Rand, J.A.; An, K.N. The influence of weakness in the vastus medialis oblique muscle on the patellofemoral joint: An in vitro biomechanical study. Clin. Biomech. 2000, 15, 335-339. [CrossRef]

25. Powers, C.M.; Chen, Y.-J.; Scher, I.S.; Lee, T.Q. Multiplane loading of the extensor mechanism alters the patellar ligament force/quadriceps force ratio. J. Biomech. Eng. 2010, 132, 024503. [CrossRef]

26. Wickiewicz, T.L.; Roy, R.R.; Powell, P.L.; Edgerton, V.R. Muscle architecture of the human lower limb. Clin. Orthop. Relat. Res. 1983, 1, 275-283. [CrossRef]

27. Sakai, N.; Luo, Z.P.; Rand, J.A.; An, K.N. Quadriceps forces and patellar motion in the anatomical model of the patellofemoral joint. Knee 1996, 3, 1-7. [CrossRef] 
28. Withrow, T.J.; Huston, L.J.; Wojtys, E.M.; Ashton-Miller, J.A. The relationship between quadriceps muscle force, knee flexion, and anterior cruciate ligament strain in an in vitro simulated jump landing. Am. J. Sports Med. 2006, 34, 269-274. [CrossRef] [PubMed]

29. Mesfar, W.; Shirazi-Adl, A. Biomechanics of the knee joint in flexion under various quadriceps forces. Knee 2005, 12, 424-434. [CrossRef] [PubMed]

30. Flaxman, T.E.; Alkjær, T.; Simonsen, E.B.; Krogsgaard, M.R.; Benoit, D.L. Predicting the functional roles of knee joint muscles from internal joint moments. Med. Sci. Sports Exerc. 2017, 49, 527-537. [CrossRef]

31. Stevens, J.E.; Mizner, R.L.; Snyder-Mackler, L. Neuromuscular electrical stimulation for quadriceps muscle strengthening after bilateral total knee arthroplasty: A case series. J. Orthop. Sports Phys. Ther. 2004, 34, 21-29. [CrossRef]

32. Moffet, H.; Collet, J.-P.; Shapiro, S.H.; Paradis, G.; Marquis, F.; Roy, L. Effectiveness of intensive rehabilitation on functional ability and quality of life after first total knee arthroplasty: A single-blind randomized controlled trial. Arch. Phys. Med. Rehabil. 2004, 85, 546-556. [CrossRef]

33. Avramidis, K.; Strike, P.W.; Taylor, P.N.; Swain, I.D. Effectiveness of electric stimulation of the vastus medialis muscle in the rehabilitation of patients after total knee arthroplasty. Arch. Phys. Med. Rehabil. 2003, 84, 1850-1853. [CrossRef]

34. Meier, W.; Mizner, R.; Marcus, R.; Dibble, L.; Peters, C.; Lastayo, P.C. Total knee arthroplasty: Muscle impairments, functional limitations, and recommended rehabilitation approaches. J. Orthop. Sports Phys. Ther. 2008, 38, 246-256. [CrossRef]

35. Kim, H.; Lee, J.; Kim, J. Electromyography-signal-based muscle fatigue assessment for knee rehabilitation monitoring systems. Biomed. Eng. Lett. 2018, 8, 345-353. [CrossRef] [PubMed]

36. Mesfar, W.; Shirazi-Adl, A. Knee joint mechanics under quadriceps-hamstrings muscle forces are influenced by tibial restraint. Clin. Biomech. 2006, 21, 841-848. [CrossRef] [PubMed]

37. Kwak, S.; Ahmad, C.; Gardner, T.; Grelsamer, R.; Henry, J.; Blankevoort, L.; Ateshian, G.; Mow, V.C. Hamstrings and iliotibial band forces affect knee kinematics and contact pattern. J. Orthop. Res. 2000, 18, 101-108. [CrossRef]

38. Bull, A.M.; Kessler, O.; Alam, M.; Amis, A.A. Changes in knee kinematics reflect the articular geometry after arthroplasty. Clin. Orthop. Relat. Res. 2008, 466, 2491-2499. [CrossRef] [PubMed]

39. Hsich, Y.-F.; Draganich, L.F. Knee kinematics and ligament lengths during physiologic levels of isometric quadriceps loads. Knee 1997, 4, 145-154. [CrossRef]

40. Zaffagnini, S.; Bignozzi, S.; Saffarini, M.; Colle, F.; Sharma, B.; Kinov, P.S.; Marcacci, M.; Dejour, D. Comparison of stability and kinematics of the natural knee versus a PS TKA with a 'third condyle'. Knee Surg. Sports Traumatol. Arthrosc. 2014, 22, 1778-1785. [CrossRef] [PubMed]

41. Wünschel, M.; Leasure, J.M.; Dalheimer, P.; Kraft, N.; Wülker, N.; Müller, O. Differences in knee joint kinematics and forces after posterior cruciate retaining and stabilized total knee arthroplasty. Knee 2013, 20, 416-421. [CrossRef]

42. Shandiz, M.A.; Boulos, P.; Saevarsson, S.K.; Yoo, S.; Miller, S.; Anglin, C. Changes in knee kinematics following total knee arthroplasty. Proc. Inst. Mech. Eng. Part H J. Eng. Med. 2016, 230, 265-278. [CrossRef] [PubMed]

43. Krackow, K.A.; Mihalko, W.M. Flexion-extension joint gap changes after lateral structure release for valgus deformity correction in total knee arthroplasty: A cadaveric study. J. Arthroplast. 1999, 14, 994-1004. [CrossRef]

44. Moynihan, A.L.; Varadarajan, K.M.; Hanson, G.R.; Park, S.-E.; Nha, K.W.; Suggs, J.F.; Johnson, T.; Li, G. In vivo knee kinematics during high flexion after a posterior-substituting total knee arthroplasty. Int. Orthop. 2010, 34, 497-503. [CrossRef] [PubMed] 